\title{
TRISSOMIA DO CROMOSSOMO 9 ASSOCIADA COM AUMENTO DA TRANSLUCÊNCIA NUCAL: CORRELAÇÃO ULTRA-SONOGRÁFICA E ANATOMOPATOLÓGICA - RELATO DE UM CASO*
}

\author{
Carlos Geraldo Viana Murta ${ }^{1}$, Paulo Roberto Merçon-de-Vargas²
}

Resumo Relatamos um caso de trissomia completa do cromossomo 9 associada com aumento da translucência nucal $(9,1 \mathrm{~mm})$, diagnosticada por ultra-som na $12^{\mathrm{a}}$ semana de gestação e confirmada por cariótipo em espécime de biópsia do vilo corial. Múltiplas anomalias congênitas foram diagnosticadas no exame ultrasonográfico e confirmadas na autópsia. Embora rara, a trissomia 9 deve ser incluída no rol das anomalias cromossômicas associadas com aumento da translucência nucal.

Unitermos: Trissomia 9. Patologia. Ultra-sonografia pré-natal. Translucência nucal. Anomalias cromossômicas.

\begin{abstract}
Trisomy 9 with increased nuchal translucency: ultrasound and pathologic correlation - a case report. We report a case of prenatal diagnosis of trisomy 9 in a fetus presenting increased translucency thickness $(9.1 \mathrm{~mm})$ observed on an ultrasound scan performed at 12 weeks pregnancy and confirmed by cariotype analysis of biopsy material obtained from the chorionic villi. Multiple trisomy 9 characteristic abnormalities were detected by ultrasound and confirmed by autopsy and histopathological examination. Although rare, trisomy 9 should be included in the list of chromosomic anomalies associated with increased translucency. Key words: Trisomy 9. Pathology. Prenatal ultrasound. Nuchal translucency. Chromosomal abnormalities.
\end{abstract}

\section{INTRODUÇÃO}

Embora as trissomias sejam as anomalias cromossômicas mais comuns em abortamentos espontâneos (52\% das cromossomopatias identificadas), somente $2 \%$ a $4 \%$ correspondem à trissomia do cromossomo $9^{(\mathbf{1}, 2)}$. A maioria dos fetos evolui para abortamento espontâneo, porém raros casos, em geral casos de mosaicismo, podem chegar ao termo. Além de restrição de crescimento, anomalias que envolvem múltiplos órgãos e sistemas têm sido descritas.

Benacerraf et al. ${ }^{(3)}$ e Chitayat et al. ${ }^{(4)}$ publicaram relatos de casos, enfatizando a possibilidade de se fazer diagnóstico ultra-sonográfico pré-natal, durante o segundo trimestre.

No presente relato, correlacionam-se as alterações ultra-sonográficas com as

* Trabalho realizado na Clínica Vitória MedifetUS e no Laboratório PAT - Anatomia Patológica, Vitória, ES.

1. Mestre pela Universidade Federal do Rio de Janeiro, Pós-graduando em Doutoramento na Universidade Federal de São Paulo/Escola Paulista de Medicina, Médico Ultrasonografista da Universidade Federal do Espírito Santo (UFES).

2. Professor Adjunto Doutor do Departamento de Patologia do Centro Biomédico da UFES. E-mail:vargaspm@zaz. com.br

Endereço para correspondência: Dr. Carlos Geraldo Viana Murta. Vitória MedifetUS. Rua das Palmeiras, 795, sala 409 Santa Lúcia. Vitória, ES, 29045-205. E-mail: cgvmurta@zaz. com.br

Aceito para publicação em 5/10/2000. anatomopatológicas de um caso de trissomia 9 completa, com ênfase nos achados passíveis de identificação ultra-sonográfica, durante o primeiro trimestre da gestação.

\section{RELATO DO CASO}

Primigesta de 30 anos de idade, cor branca, com data da última menstruação em 1/5/98, sem doenças pregressas significativas.

Um exame ultra-sonográfico na $11^{\mathrm{a}}$ semana pós-menstrual revelou gravidez única, com saco gestacional normo-implantado, contendo feto viável com freqüência cardíaca de 161 bpm, comprimento cabeça-nádegas $(\mathrm{CCN})$ de $41 \mathrm{~mm}$, compatível com a $11^{\mathrm{a}}$ semana pós-menstrual, e medida da translucência nucal (TN) de $6 \mathrm{~mm}$. Na $13^{\mathrm{a}}$ semana, novo exame ultra-sonográfico mostrou feto viável, com CCN de $57 \mathrm{~mm}$ (restrição do crescimento), TN de 9,1 $\mathrm{mm}$ de espessura, foco ecogênico intracardíaco, imagem compatível com defeito do septo interventricular, pelve renal dilatada bilateralmente e intestino hiperecogênico (Figura 1).

A Dopplerfluxometria revelou alterações no retorno cardíaco, denotando insuficiência cardíaca fetal. A análise ci- togenética de amostra obtida por biópsia de vilo corial concluiu por trissomia completa do cromossomo 9 . Na $14^{\mathrm{a}}$ semana, ocorreu óbito fetal, seguido de eliminação dez dias após o óbito.

O estudo anatomopatológico mostrou feto do sexo masculino, macerado grau III, com múltiplas anomalias (Figura 2): hipertelorismo ocular, pavilhões auriculares pequenos (microtia) e com implantação baixa, fenda labial unilateral esquerda, arco palatino elevado (em arco gótico) com fenda incompleta, comunicação interventricular septal ampla, ausência de fixação dos intestinos delgado e grosso e discreta ectasia da pelve e cálices renais bilateralmente. À parte implantação do cordão umbilical paralela à placa corial, não foram observadas alterações nos anexos fetais. O exame histopatológico revelou vilosidades coriônicas com edema difuso sem cisternas e fibroelastose endocárdica no ventrículo esquerdo. As demais vísceras não mostraram anormalidades histológicas apreciáveis.

\section{DISCUSSÃO}

O aprimoramento da qualidade dos aparelhos de ultra-sonografia, especialmente o ultra-som vaginal com transdu- 

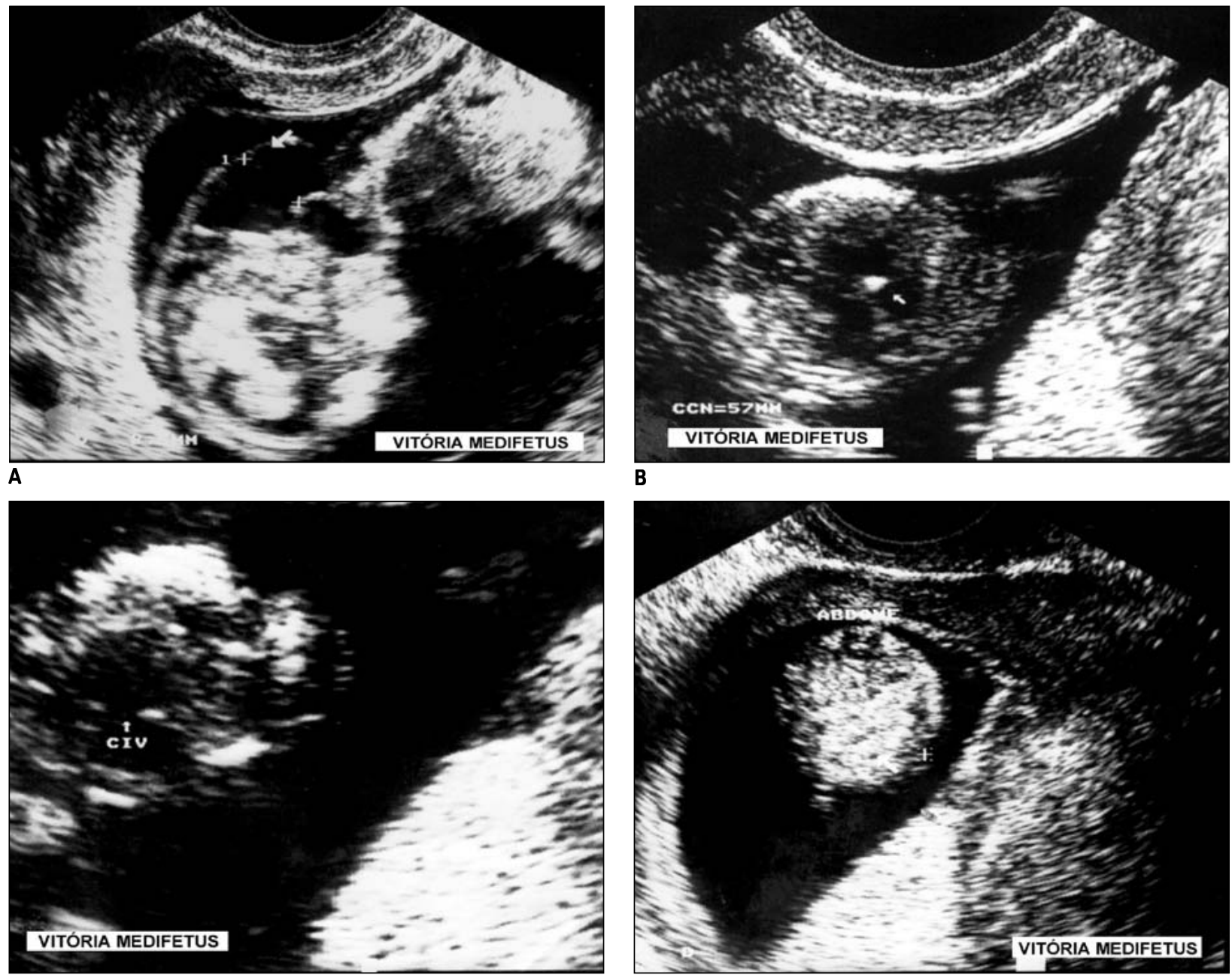

B

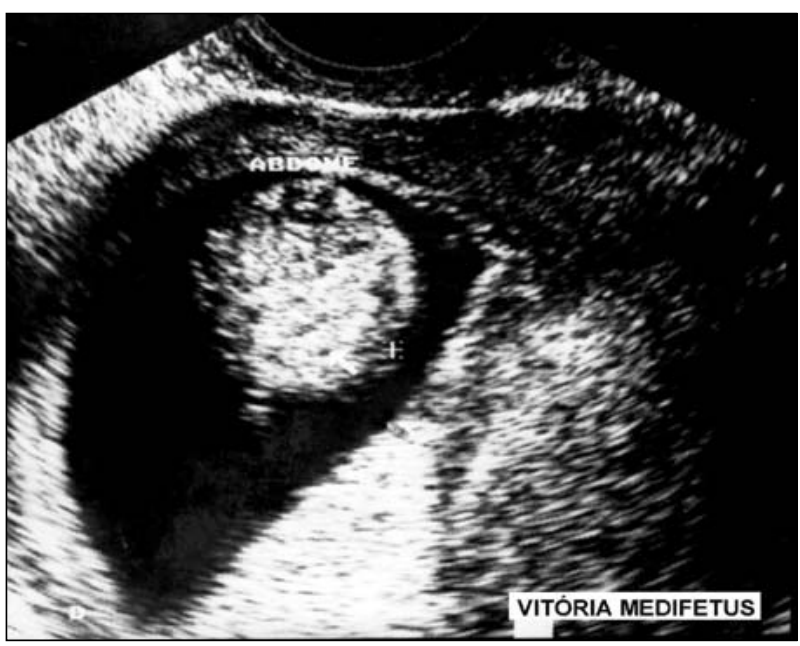

C

D

Figura 1. Ultra-som de 12 semanas de gestação mostrando: translucência nucal de 9,1 mm (A); foco ecogênico intracardíaco (B); comunicação interventricular (C); hiperecogenicidade do intestino (D); pieloectasia renal, bilateralmente $(\mathbf{E})$.

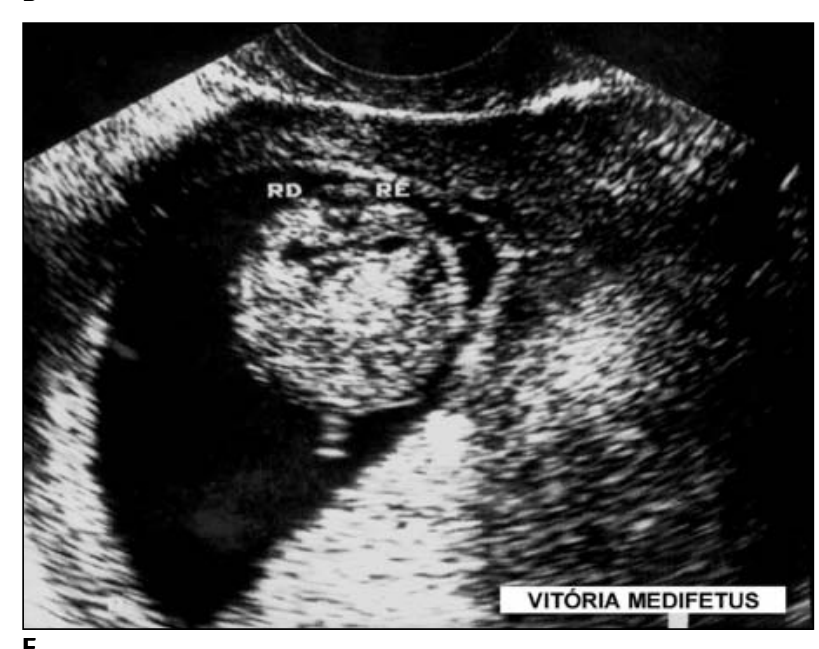

tor de alta freqüência, tem possibilitado o estudo da anatomia fetal e o diagnóstico de anomalias congênitas já no primeiro trimestre de gestação.

Dentre os vários marcadores diagnósticos empregados no rastreio de anoma- lias cromossômicas, destaca-se a medida da TN entre 10 e 14 semanas de gestação ${ }^{(5)}$. Uma grande proporção de fetos portadores de trissomias dos cromossomos 21,18 e 13 , de monossomia $X$ e de triploidias apresentam medida da $\mathrm{TN}$ maior que $3 \mathrm{~mm}$. O aumento da $\mathrm{TN}$ tem sido atribuído a distúrbio hemodinâmico do feto (insuficiência) decorrente de defeitos da anatomia do coração ou dos grandes vasos ${ }^{(6)}$. Como as anomalias cardíacas ocorrem em aproximadamente 

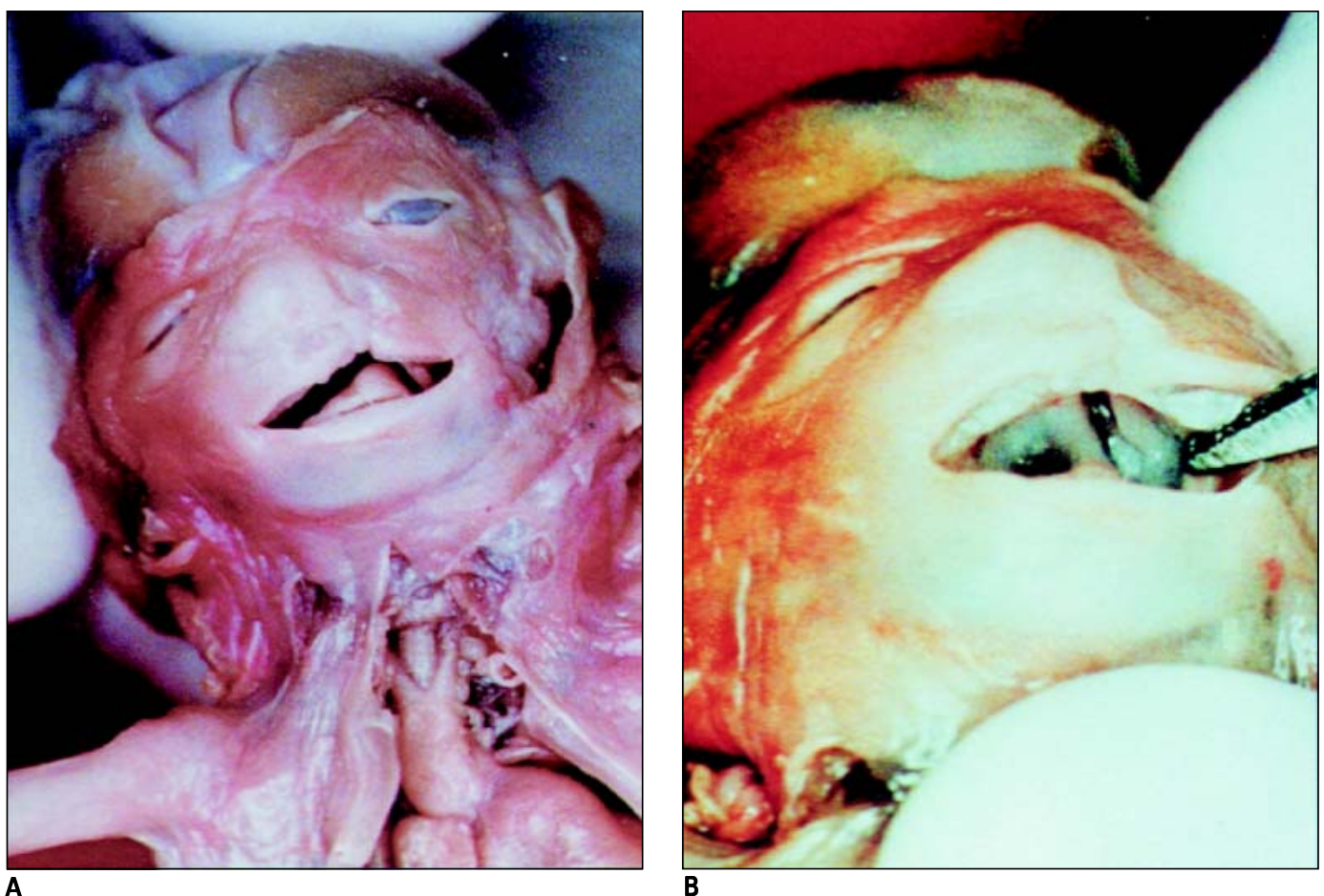

A

B
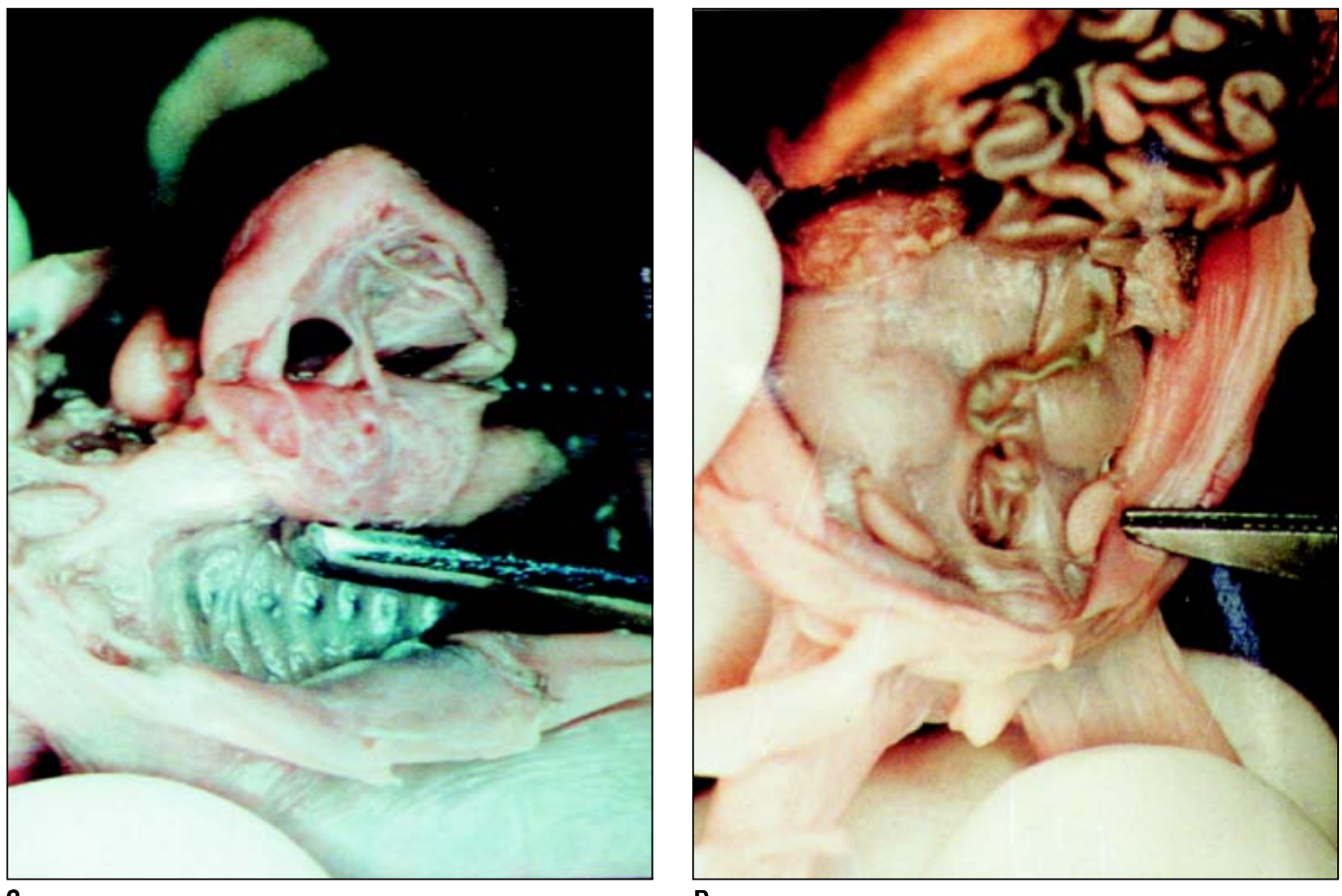

Figura 2. Fotomacrografias mostrando: múltiplas anomalias faciais - hipertelorismo, fenda labial e implantação baixa do pavilhão auricular (A); fenda palatina (B); comunicação interventricular (C); ausência de fixação intestinal (D).

dois terços dos casos de trissomia $9^{(7)}$, é de se esperar que o aumento da TN constitua um marcador também para esta cromossomopatia.

O fenótipo da trissomia do cromossomo 9 não se diferencia daquele de ou- tras trissomias, especialmente das trissomias 13 e 18, sendo indispensável o estudo citogenético. Dentre as numerosas anomalias descritas, são comuns, de acordo com Gilbert-Barness ${ }^{(\mathbf{8})}$ : microcefalia ou dolicocefalia, assimetria facial, hipo ou hipertelorismo ocular, fissura palpebral pequena, prega epicântica, fenda palatina e labial, achatamento da base do nariz, micrognatia, microstomia, arco palatino elevado (em arco gótico), fenda labial e/ou palatina, pavilhões auri- 
culares deformados e/ou com implantação baixa, implantação baixa do couro cabeludo, pescoço curto ou alado, hipertelorismo mamário, hérnia umbilical, criptorquidia, micropênis, cifose ou escoliose, agenesia do púbis, agenesia ou hipoplasia da tíbia ou fíbula, agenesia ou deformação dos pododáctilos, anomalias cardíacas, cisto renais, hidronefrose e dilatação do IV ventrículo cerebral. Merece realce que o diagnóstico foi suspeitado, e a biópsia de vilo corial foi indicada na $12^{\mathrm{a}}$ semana pós-menstrual, pelos achados ultra-sonográficos de grande aumento da TN (9,1 mm), dilatação da pelve renal, hiperecogenicidade intestinal, sinal do "golf ball", comunicação interventricular e restrição precoce do crescimento fetal.

Segundo Murta ${ }^{(9)}$, o foco ecogênico intracardíaco (sinal do "golf ball") é uma representação da mineralização do músculo papilar. A importância deste sinal reside na sua freqüente associação com as trissomias 21,13 e 18 , a monossomia $\mathrm{X}$, a triploidia e as translocações não-balanceadas ${ }^{(\mathbf{9 , 1 0})}$. A associação com trissomia 9 ainda não havia sido relatada. No presente caso, não havia necrose ou calcificação miocárdica mas fibroe- lastose, que julgamos poder também explicar o foco hiperecogênico.

A hiperecogenicidade dos intestinos e a pieloectasia renal são marcadores sonográficos reconhecidos para a síndrome de Down no segundo trimestre da gestação $^{\mathbf{1 1 1}, 12)}$, mas a presença dessas anomalias em caso de trissomia 9 ainda não havia sido relatada. Em nosso entendimento, o aumento da ecogenicidade intestinal deve-se à concentração das alças intestinais na porção central do abdome, decorrente de sua não-fixação.

$\mathrm{O}$ presente relato demonstra o valor do exame ultra-sonográfico para a detecção de anormalidades marcadoras de anomalias cromossômicas, em especial indicando que se deve incluir a trissomia 9 no rol das anomalias cromossômicas associadas com o aumento da TN, comunicação interventricular, sinal de "golf ball" e hiperecogenicidade intestinal.

\section{REFERÊNCIAS}

1. Boué J, Boué A, Lazar P. Retrospective and prospective epidemiological studies of 1500 karyotypes from spontaneous human abortions. Teratology 1975;12:11-26.

2. Lauritsen JG. The cytogenetics of spontaneous abortion. Res Reprod 1982;14:3-4.
3. Benacerraf BR, Pauker S, Quade BJ, Bieber FR. Prenatal sonography in trisomy 9. Prenat Diagn 1992;12:175-81.

4. Chitayat D, Hodgkinson K, Luke A, Winsor E, Rose T, Kalousek D. Prenatal diagnosis and fetopathological findings in five fetuses with trisomy 9. Am J Med Genet 1995;56:247-51.

5. Snijders RJM, Noble P, Sebeire N, Souka A, Nicolaides KH. UK multicentre project on assessment of risk of trisomy 21 by maternal age and fetal nuchal translucency thickness at 10-14 weeks of gestation. Fetal Medicine Foundation First Trimester Screening Group. Lancet 1998;352: 343-6.

6. Murta CGV, Moron AF, Ávila MAP, França LC. Alterações cromossômicas e cardíacas: novas perspectivas com o emprego do Doppler colorido. Radiol Bras 1999;32:169-73.

7. Kurnick J, Atkins L, Feingold M, Hills J, Dvorak A. Trisomy 9: predominance of cardiovascular, liver, brain, and skeletal anomalies in the first diagnosed case. Hum Pathol 1974;5:223-32.

8. Gilbert-Barness E. Chromosomal abnormalities. In: Gilbert-Barness, E, ed. Potter's Pathology of the fetus and infant. St. Louis: Mosby: 1996;388432.

9. Murta CGV. Foco ecogênico no coração fetal: importância clínica. Femina 2000;28:253-5.

10. Roberts DJ, Genest D. Cardiac histologic pathology characteristic of trisomies 13 and 21. Hum Pathol 1992;23:1130-40.

11. Benacerraf BR. The second trimester fetus with Down syndrome: detection using sonographic features. Ultrasound Obstet Gynecol 1996;7:14755.

12. Benacerraf BR, Barss VA, Laboda LA. A sonographic sign for the detection in the second trimester of the fetus with Down's syndrome. Am J Obstet Gynecol 1985;151:1078-9. 